\title{
Sistematização da assistência de enfermagem: Um relato de caso
}

\author{
Systematization of nursing care: A care report \\ Sistematizacón de la atención de enfermeira: Reporte de um caso
}

Júlia Sangalli Demichei

ORCID: https://orcid.org/0000-0001-8590-7231 Universidade do Vale do Taquari, Brasil

E-mail: julia.demichei@universo.univates.br

Laura Marchetti Gritti

ORCID: https://orcid.org/0000-0002-2864-8605 Universidade do Vale do Taquari, Brasil E-mail: laura.gritti@universo.univates.br

Arlete Eli Kunz da Costa

ORCID: https://orcid.org/0000-0002-5655-3646 Universidade do Vale do Taquari, Brasil

E-mail: arlete.costa@univates.br

Paula Michele Lohmann

ORCID: https://orcid.org/0000-0002-8429-9155 Universidade do Vale do Taquari, Brasil

E-mail: paulalohmann@univates.br

\begin{abstract}
Resumo
Trata-se de um relato de caso, desenvolvido por duas acadêmicas do curso de Enfermagem de uma instituição localizada no interior do estado do Rio Grande do Sul/Brasil. O objetivo do estudo é discutir a Sistematização da Assistência de Enfermagem (SAE) aplicada em uma paciente portadora do diagnóstico médico de Fratura na Coluna em L3. A realização do trabalho ocorreu na disciplina de Enfermagem na saúde do adulto e idoso, durante o primeiro semestre de 2020. Após a coleta de dados e escuta da paciente, realizou-se a elaboração de diagnósticos de enfermagem utilizando a taxonomia NANDA e intervenções de enfermagem por meio da NIC, tendo como objetivo contribuir na prática profissional e no prognóstico da paciente, com o intuito de proporcionar melhora na qualidade de vida e bem-estar da paciente por meio de ações e intervenções de enfermagem. Após a realização do estudo de caso e experiência das acadêmicas, pode-se concluir a importância da aplicação da SAE, a qual tem como objetivo alcançar a melhora do quadro clínico da paciente, por meio de intervenções, cada qual com sua importância.
\end{abstract}

Palavras-chave: Processo de enfermagem; Currículo; Ensino; Enfermeiro; Estudo.

\begin{abstract}
This is a case report, developed by two nursing students from an institution located in the interior of the state of Rio Grande do Sul / Brazil. The objective of the study is to discuss the Nursing Care Systematization (SAE) applied to a patient with a medical diagnosis of Spine Fracture in L3. The work was carried out in the discipline of Nursing in adult and elderly health, during the first semester of 2020. After collecting data and listening to the patient, nursing diagnoses were elaborated using the NANDA taxonomy and nursing interventions. through the NIC, aiming to contribute to the patient's professional practice and prognosis, with the aim of providing improvement in the patient's quality of life and well-being through nursing actions and interventions. After conducting the case study and experience of the academics, it is possible to conclude the importance of the application of SAE, which aims to achieve the improvement of the patient's clinical condition, through interventions, each with its importance.
\end{abstract}

Keywords: Nursing process; Curriculum; Teaching; Nurse; Study.

\section{Resumen}

Se trata de un caso clínico, desarrollado por dos estudiantes de enfermería de una institución ubicada en el interior del estado de Rio Grande do Sul / Brasil. El objetivo del estudio es discutir la Sistematización de la Atención de Enfermería (SAE) aplicada a un paciente con diagnóstico médico de Fractura de Columna Vertebral en L3. El trabajo se realizó en la disciplina de Enfermería en salud de adultos y ancianos, durante el primer semestre de 2020. Luego de recolectar datos y escuchar al paciente, se elaboraron diagnósticos de enfermería utilizando la taxonomía NANDA e intervenciones de enfermería a través de la NIC, con el objetivo de Contribuir a la práctica profesional y al pronóstico del paciente, con el objetivo de proporcionar una mejora en la calidad de vida y el bienestar del paciente a través de acciones e intervenciones de enfermería. Luego de realizar el estudio de caso y la experiencia de los académicos, es posible concluir la importancia de la aplicación de SAE, que tiene como objetivo lograr la mejora de la condición clínica del paciente, a través de intervenciones, cada una con su importancia.

Palabras clave: Proceso de enfermería; Plan de estudios; Enseñando; Enfermera; Estudiar. 


\section{Introdução}

As fraturas ocorridas na região lombar da coluna, entre as vértebras L1 e L12, são bastante comumente em pessoas idosas, com diagnóstico de osteoporose - doença óssea progressiva, que causa o enfraquecimento dos ossos. A osteoporose em um contexto geral é a causadora de muitas quedas e fraturas em pessoas mais envelhecidas, em outras pessoas da faixa etária adulta e juventude as lesões lombares ocorrem geralmente devido a traumas de alta energia, acidentes de trânsito, desportivo ou violências, entre outros. É importante evidenciar que as fraturas nesta região podem ser de alto risco, causando danos na medula espinhal e frequentemente causando danos nos nervos da medula ou do cérebro (Sobrice, 2017).

No contexto acima, conforme a Resolução 358/2009 (Cofen, 2009) o enfermeiro atua na Sistematização da Assistência de Enfermagem (SAE), qual é dividido por cinco etapas: histórico (anamnese e exame físico), diagnóstico de enfermagem (manual do diagnóstico de enfermagem), prescrição/intervenção, planejamento e avaliação de enfermagem. Para realização desta sistematização o enfermeiro utiliza as taxonomias NANDA (North American Nursing Diagnosis Association), NIC (Nursing Interventions Classification) e NOC (Nursing Outcomes Classification), referências mundialmente reconhecidas e aplicadas para todos os âmbitos de avaliação de enfermagem.

Portanto, este estudo tem o objetivo de discutir a Sistematização da Assistência de Enfermagem (SAE) aplicada em uma paciente portadora do diagnóstico médico de Fratura na Coluna em L3, além de formalizar a experiência das estudantes no desenvolvimento da Sistematização da Assistência de Enfermagem.

\section{Metodologia}

Trata-se de um estudo de caso realizado por meio de uma experiência na aplicação do Processo de Enfermagem em um familiar durante a disciplina de Saúde do Adulto e Idoso I. Os dados para a implantação da sistematização foram coletados nos meses de fevereiro e março de 2020, por meio da vivência e visita domiciliar das acadêmicas. Para a montagem da sistematização foram aplicados os seguintes quesitos: exame físico céfalo-caudal e realização de entrevista com paciente e familiares adquirindo um histórico completo. Em seguida, através do NANDA (North American Nursing Diagnosis Association) e NIC (Nursing Interventions Classification) foram elencados os diagnósticos e as intervenções adequadas para o caso. E por fim após todos estes dados registrados foi feita a avaliação final da paciente por meio da NOC (Nursing Outcomes Classification)

Este estudo de caso é classificado como pesquisa qualitativa, pois permite que se possa investigar o indivíduo com profundidade, analisando variáveis importantes, como: desenvolvimento do objeto de estudo, planejamento de cuidados a serem propostos e evolução do caso (Bocchi, Meneguin, \& Santi, 1996).

\section{Resultados e Discussão}

A paciente E.R.M. 83 anos, sexo feminino, branca, brasileira, viúva, aposentada, católica, cinco filhos, residia sozinha na Cidade de Encantado, tendo cuidadora 24h. Histórico de fratura na Coluna em L3 recente, osteoporose, hipertensão e depressão. Em 2016 a paciente caiu e fraturou o fêmur e posteriormente foi realizada cirurgia de colocação de prótese. Em 2019, a mesma teve outra queda, ocasionando fratura no rádio e na ulna.

No mês de fevereiro de 2020 a paciente procurou o hospital devido a fortes dores na região lombo-sacral, o médico atendente avaliou e à diagnosticou com nervo ciático inflamado, medicou-a com analgésicos e em seguida a liberou. Na semana seguinte, ainda com bastante dor, a paciente retornou ao hospital referindo algia intensa em dorso e membros inferiores, falta de ar, náuseas, constipação, afagia, insônia e desânimo. O médico solicitou um Raio X, constatando uma Fratura na Coluna em L3, orientando a paciente a usar colete Jewett. Ao passar dos dias seu quadro clínico decaiu, com dores 
mais intensas, dispneias e sudoreses. Frente estes episódios de mal-estar da paciente, a família optou por procurar um especialista, que recomendou o uso de colete Putti, mais adequado para o diagnóstico.

Os medicamentos utilizados pela paciente são: olmelsartana medoxomila + hidroclotiazida $40 \mathrm{mg}+25 \mathrm{mg}, 1 \mathrm{cp} 1 \mathrm{x}$ ao dia, succinato de metoprolol $50 \mathrm{mg}, 1 \mathrm{cp} \mathrm{1x}$ ao dia, cloridrato de duloxitina $60 \mathrm{mg}, 1 \mathrm{cp} \mathrm{1x}$ ao dia, cloridrato de cálcio + colecalciferol $1500 \mathrm{mg}, 1 \mathrm{cp} 1 \mathrm{x}$ ao dia, alprazolam $0,5 \mathrm{mg} 1 \mathrm{cp} 1 \mathrm{x}$ ao dia antes de dormir, pregabalina $1 \mathrm{cp} 1 \mathrm{x}$ ao dia por 5 dias a noite, e cloridrato de oxicodona $1 \mathrm{cp} 1 \mathrm{x}$ a noite.

Ao exame físico, observou-se que a paciente se encontrava descorada, com membros inferiores e superiores com baixa perfusão, acamada, inquieta, inapetente, dispnéica, pele seca, uso de colete para fratura e início de úlcera de pressão em cotovelos. Sinais vitais: PA 130/60 mmHg, FR 29 mpm, FC 125 bpm, To ax 37 C, sat 90\%.

De acordo com a história da paciente e o relato familiar, foram estabelecidos diagnósticos de enfermagem juntamente com as intervenções, conforme descrito no Quadro 1.

Quadro 1. Diagnósticos e Intervenções de Enfermagem prestados a paciente.

\begin{tabular}{|c|c|c|}
\hline Diagnósticos de Enfermagem & Resultado esperado & Intervenções de Enfermagem \\
\hline Náuseas & $\begin{array}{l}\text { Melhora no quadro relacionado a } \\
\text { náuseas }\end{array}$ & - Medicar conforme prescrição médica \\
\hline $\begin{array}{l}\text { Incapacidade para manter a } \\
\text { respiração }\end{array}$ & Melhora no quadro respiratório & $\begin{array}{ll}- & \text { Instalar } \mathrm{O} 2 \\
\text { - } & \text { Manter cabeceira elevada a } 45^{\circ}\end{array}$ \\
\hline Padrão respiratório ineficaz & Melhora no quadro respiratório & $\begin{array}{ll}\text { - } & \text { Observar respiração } \\
\text { - } & \text { Manter cabeceira da cama elevada }\end{array}$ \\
\hline Deglutição prejudicada & $\begin{array}{l}\text { Melhora no quadro clínico, } \\
\text { possibilitando alimentação } \\
\text { equilibrada e rica em nutrientes. }\end{array}$ & $\begin{array}{ll}\text { - } & \text { Posicionar paciente de forma adequada } \\
\text { (cabeceira elevada) } \\
\text { - Incentivar paciente a se alimentar } \\
\text { - } \quad \text { Sugerir líquidos }\end{array}$ \\
\hline $\begin{array}{l}\text { Alteração na nutrição: menos } \\
\text { que o corpo necessita }\end{array}$ & $\begin{array}{l}\text { Proporcionar bem-estar e qualidade } \\
\text { de vida, fazendo com que o indivíduo } \\
\text { ingira nutrientes suficientes para } \\
\text { sustentação do organismo; }\end{array}$ & $\begin{array}{l}\text { - Controle da nutrição conforme } \\
\text { orientações nutricionais; }\end{array}$ \\
\hline Mobilidade física prejudicada & Promover auxílio na locomoção; & $\begin{array}{l}\text { - Orientar acompanhante para auxiliar a } \\
\text { paciente se locomover; }\end{array}$ \\
\hline Integridade da pele prejudicada & $\begin{array}{l}\text { Melhorar o estado físico da pele, } \\
\text { proporcionando conforto e bem-estar } \\
\text { a paciente; }\end{array}$ & $\begin{array}{l}\text { - Controle da ingestão hídrica conforme } \\
\text { orientações nutricionais }\end{array}$ \\
\hline $\begin{array}{l}\text { Déficit no autocuidado: } \\
\text { alimentação, banho e higiene, } \\
\text { vestir-se/arrumar-se }\end{array}$ & $\begin{array}{l}\text { Proporcionar auxílio na higiene e } \\
\text { banho garantindo melhor qualidade } \\
\text { de vida; }\end{array}$ & $\begin{array}{l}\text { - Proporcionar bem-estar e ânimo a } \\
\text { paciente; } \\
\text { - } \quad \text { Garantir a boa higiene; }\end{array}$ \\
\hline Impotência & $\begin{array}{l}\text { Promover autoconfiança, objetivando } \\
\text { melhora no seu quadro }\end{array}$ & $\begin{array}{l}\text { - Apoiar as práticas espirituais da } \\
\text { paciente } \\
\text { - } \quad \text { Proporcionar conversas }\end{array}$ \\
\hline Distúrbio no padrão do sono & $\begin{array}{l}\text { Melhorar a qualidade do sono da } \\
\text { paciente, proporcionando noites bem } \\
\text { dormidas e saúde mental preservada }\end{array}$ & $\begin{array}{l}\text { - } \quad \text { Proporcionar um ambiente calmo } \\
\text { - } \quad \text { Garantir que a paciente durma ao menos } \\
\text { 6h corridas }\end{array}$ \\
\hline
\end{tabular}




\begin{tabular}{|l|l|l|}
\hline Alteração na perfusão tissular & Sinais vitais estáveis; & $\begin{array}{l}- \text { Controlar os sinais vitais } \\
\text { Medicar conforme prescrição médica }\end{array}$ \\
\hline Constipação & $\begin{array}{l}\text { Melhora no quadro digestivo da } \\
\text { paciente, tendo em vista o bom } \\
\text { funcionamento e a normalidade } \\
\text { corpórea }\end{array}$ & $\begin{array}{l}\text { Controle da nutrição através de } \\
\text { orientações nutricionais; }\end{array}$ \\
$\begin{array}{l}\text { Controle da ingestão hídrica por meio } \\
\text { de orientações nutricionais; } \\
\text { Observar eliminações fisiológicas }\end{array}$ \\
\hline
\end{tabular}

Fonte: Do autor (2020).

Considerando os diagnósticos e as intervenções citadas acima foram planejadas e propostas algumas medidas para a paciente, com auxílio da família, cuidadores e equipe de enfermagem que acompanhará durante este período de internação. Quanto a incapacidade de manter a respiração, foi instalado na paciente óculos nasal a 2 mg por minuto de O2, e solicitado que a cabeceira se mantenha elevada para melhora no quadro respiratório. Quanto a ingestão hídrica e à alimentação menos que o organismo necessita, foi solicitado uma nutricionista para acompanhamento da mesma, e orientado aos familiares e cuidadores para que insistam na ingestão hídrica e na ingestão de alimentos protéicos e pastosos, já que a idosa possui grande resistência para alimentar-se adequadamente. Por tratar-se de uma paciente acamada é importante grande atenção nos quesitos hídricos e alimentares para que se evite formação de escaras e para que o diagnóstico de constipação da paciente volte a sua origem de funcionalidade.

Quanto ao diagnóstico de mobilidade física prejudicada, correlacionado à fratura lombar em L3, e a intensa dor referida pela paciente, solicita-se que a mesma se mantenha em repouso, fazendo uso correto do colete indicado pela equipe médica e mantendo o uso dos analgésicos prescritos. Ressalta-se atenção para o quadro de dor, chamando equipe médica se necessário para reavaliação de analgésicos. No que se tange ao déficit do autocuidado, este, pode encontrar-se prejudicado devido a impossibilidade da autonomia da paciente. O diagnóstico de distúrbio do padrão de sono, está relacionado a intensa dor referida pela paciente, o que a impossibilita de descansar tranquilamente e ter uma boa noite de sono.

Conforme a vivência citada, realizada durante a desenvoltura da disciplina, conseguimos elaborar um plano de cuidado para a paciente, com visão à melhora no quadro clínico, implantando a SAE, conforme aprendido e estudado em aula. O planejamento e as medidas foram propostos, porém a paciente decaiu rapidamente, devido ao seu quadro respiratório, o que a impossibilitou de aderir ao tratamento conforme proposto. Durante todo processo vivenciado, conseguimos entender a importância do papel do enfermeiro nos cuidados à pacientes com fratura em L3, compreendemos o quão importante é os cuidados à um paciente acamado, buscando sempre prevenir a formação de escaras. Salienta-se ainda o apoio e as orientações que o enfermeiro deve passar ao paciente e familiares, que é de suma importância, suprindo as dúvidas referentes aos cuidados com esta patologia, estimulando sempre para a melhora.

Quanto ao atendimento prestado à pacientes com fraturas lombares, deve-se levar em consideração que este, até então tratava-se de uma pessoa ativa, com total autonomia, salvo algumas exceções. No entanto, o enfermeiro deve prestar uma atuação efetiva e segura, avaliando frequentemente o estado do paciente, evitando complicações futuras, pois, apesar de tratarse de fraturas de nível simples, pode vir a acontecer complicações que atinjam o funcionamento geral do corpo do/a paciente (Comarú, 1975).

Nesses casos, o enfermeiro atua aplicando seus conhecimentos gerais, sempre zelando pela saúde do paciente, aplicando medidas de urgência para a melhora do quadro e da região afetada (Idem). 


\section{Considerações Finais}

Após a realização do estudo de caso e experiência das acadêmicas, pode-se concluir a importância da aplicação da SAE, a qual tem como objetivo alcançar a melhora do quadro clínico da paciente, por meio de intervenções, cada qual com sua importância. Durante o desenvolvimento deste processo percebe-se a importância do acompanhamento, que se faz essencial para a elaboração da prescrição e intervenções de enfermagem.

Apesar de todas intervenções propostas para a evolução do quadro clínico, a paciente decaiu rapidamente devido a uma pneumonia, o que a levou a óbito em poucos dias. Tendo em vista estes acontecimentos ressaltamos que é de suma importância que os enfermeiros estejam capacitados e habilitados para o manejo de pacientes com fraturas, para assim, prestarem $o$ atendimento adequado.

Salienta-se ainda sobre a importância de os estudantes vivenciarem estas realidades durante a formação acadêmica, podendo assim relacionar a teoria e prática, buscando intervenções atreladas as teorias aprendidas.

\section{Referências}

Bocchi, S. C. M., Meneguin, S., \& Santi, R. C. (1996). Sistematização da Assistência de Enfermagem a paciente com luxação de coluna cervical: estudo de caso. Revista Latino-Americana de Enfermagem, 4(2). Recuperado de https://www.scielo.br/scielo.php?pid=S0104-11691996000200009\&script=sci_arttext. $\quad$ doi: $\quad$ 10.1590/S010411691996000200009

Comarú, M. N. (1975). Atuação da enfermeira na unidade de traumatologia. Revista Brasileira de Enfermagem, 28 (2), 81-87. doi: 10.1590/0034-716719750002000013

Conselho Federal de Enfermagem (Cofen). (2009). Resolução COFEN-358/2009. Recuperado em 18 de maio de 2020, de http://www.cofen.gov.br/resoluo-cofen-3582009_4384.html

Herdman, T., \& Shigemi, H. K. (2018). Diagnósticos de enfermagem da NANDA-I: definições e classificações 2018-2020. Porto Alegre: Artmed.

Sociedade Brasileira de Radiologia Intervencionista e Cirurgia Endovascular (Sobrice). (2017). Folheto informativo. Recuperado em 18 de maio de 2020, de http://www.sobrice.org.br/paciente/condicoes-clinicas/fratura-vertebral 